\title{
Replacement of the mitral valve with autologous fascia lata
}

\author{
Michael Petch, Jane Somerville, Donald Ross, Keith Ross, Richard Emanuel, and Lawson \\ McDonald \\ From the Cardiothoracic Institute and National Heart Hospital, London
}

The results of replacement of the mitral valve with frame mounted autologous fascia lata in 48 patients have been examined 2 to 4 years after operation. There were 7 early deaths and 7 late deaths. Five patients had reoperation for mitral regurgitation and an additional 9 are unwell and will probably need further surgery. Thirty-seven ( $90 \%$ ) of the survivors left hospital with a competent valve. Only 2 remain so after an average follow-up period of 43.9 months. The natural history of mitral fascia lata valves is slow deterioration frequently causing regurgitation within 12 to 18 months. There is progressive thickening and stiffening of the cusps, with retraction of the posterior cusp resulting in severe regurgitation with some obstruction. It is concluded that this form of mitral valve replacement is not satisfactory after I to 2 years.

Replacement of heart valves may be the only satisfactory form of therapy for patients with severe valvar disease, but the ideal substitute has yet to be found. Following the report by Senning (1967) on the use of autologous fascia lata, this tissue has been used for aortic and mitral valvar replacement. The early results looked promising (Ionescu and Ross, 1969), but longer experience with supported fascia lata valves in the mitral position has shown that this is not a satisfactory form of valve replacement (McEnany, Ross, and Yates, 1972 ; Ross, GonzalezLavin, and Dalichau, 1962). This paper reports the results three to four years after the operation.

\section{Subjects and method}

Forty-eight patients had replacement of the mitral valve with autologous fascia lata at the National Heart Hospital between May 1969 and September 1970. The survivors were followed up to July I973, with a minimum followup period of 2 years and 10 months and a maximum of 4 years. There were 33 women and 15 men, aged 19 to 64 years (mean age 44.7 years). The aetiology of the valvar disease was rheumatic in 44, infective endocarditis in 2 , and rupture of the chordae tendineae in 2 , one of whom had coexistent coronary heart disease.

All the patients were severely incapacitated and 2 required emergency valvar replacement. Twelve patients had had one previous mitral valvotomy and 3 had had two Received 6 August 1973

1 This work was supported by a grant from the British Heart Foundation to J.S. until I April I973 and continued support has been given by the Clinical Research Committee Funds of the National Heart and Chest Hospitals. previous mitral operations. 36 patients were in atrial fibrillation and 5 of them had previous systemic emboli. Preoperative cardiac catheterization was performed in $\mathbf{3 7}$ patients; the resting pulmonary arterial pressure exceeded $60 \mathrm{mmHg}$ in 17 and was normal in only I patient. All patients had radiographic evidence of cardiomegaly, with left atrial enlargement and pulmonary venous congestion. The majority had a long history of slow but progressive deterioration and were accepted for operation with the knowledge that they formed a high risk group.

The surgical technique has previously been described in detail (Ionescu et al., 1970). The valves were prepared at the time of operation and mounted on a metal frame in 45 and a polypropylene support in 3. Additional surgical procedures performed were aortic valvar replacement with a Starr valve in 2 and an aortic homograft in 4, tricuspid annuloplasty in 5, and tricuspid valvotomy in $\mathbf{I}$.

Since operation all surviving patients have been assessed regularly in outpatients by clinical examination, electrocardiography, and chest radiography. Digoxin has been continued in all and diuretics in 31 out of 34 survivors; anticoagulant therapy has been continued in II at the discretion of the physician in charge.

Twenty-two patients had postoperative cardiac catheterization at I to 33 months after operation. In II it was done between 6 and 18 months and in 2 it was done in the first month after operation.

\section{Early mortality \\ Results}

There were 7 deaths within 4 weeks of operation including I patient who had an emergency operation and died in the operating theatre and 2 who died with low cardiac output shortly after operation. 
One of these patients in shock had 48 minutes of ischaemic arrest and the other had a cardiac infarction during operation. A further patient had a cerebrovascular accident during operation and died of pulmonary complications. The fifth patient died 5 weeks after operation from myocardial failure after haemorrhage and bouts of ventricular failure - the mitral valve was competent. One patient died from multiple systemic emboli from fragmentation of a left atrial thrombus. The final patient died 3 weeks after a difficult operation which also involved closing an aorto-atrial fistula; her postoperative course was complicated by heart block, multiple pulmonary and systemic emboli, and a haemolytic anaemia. Mitral valvar dysfunction did not contribute to early deaths.

\section{Late deaths}

There were 7 late deaths occurring after patients had left hospital; 2 were due to pulmonary embolism at 7 and 21 months, respectively; $I$ of these patients was on treatment with anticoagulants but the thrombotest was inadequately controlled. One death was due to hepatitis 4 months after operation, I to congestive cardiac failure at 19 months despite only trivial mitral regurgitation but this patient had had a long period of ischaemic arrest during sur-

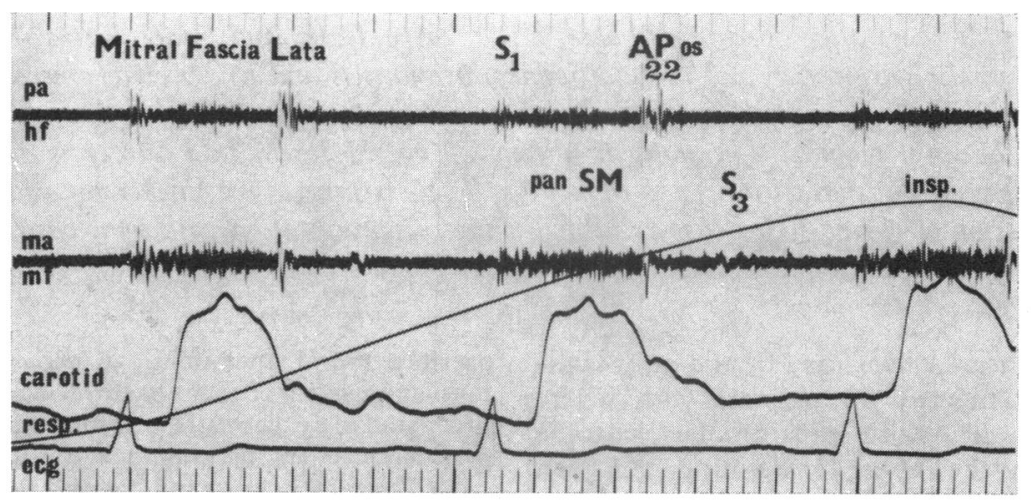

$a$

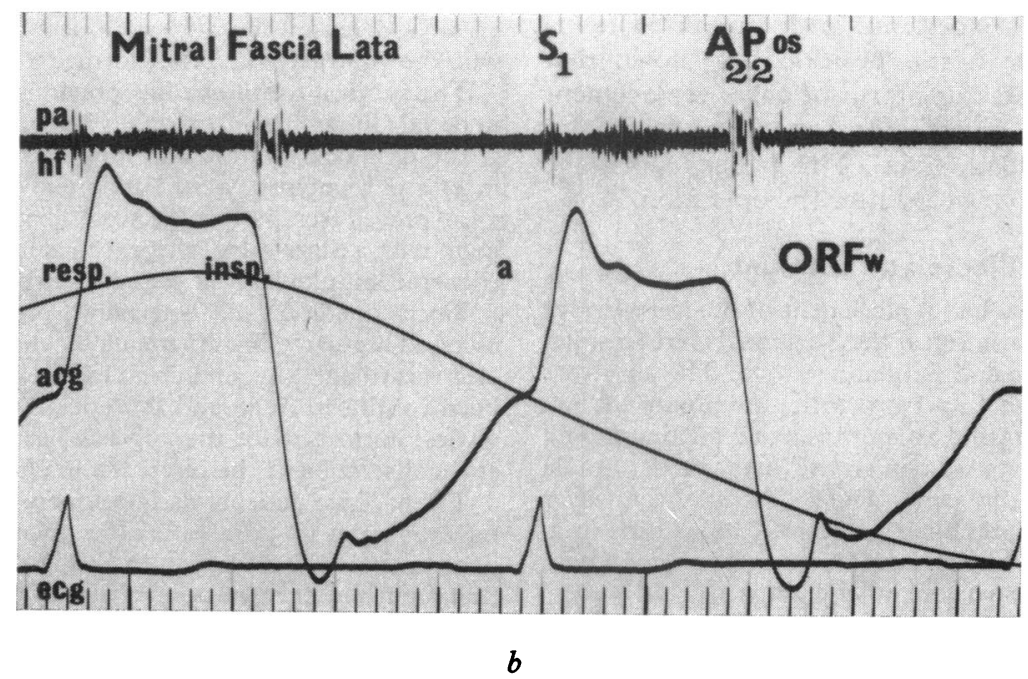

FIG. I The characteristic phonocardiographic features of a mitral fascia lata valve. (a) High frequency recording over pulmonary area (pa $h f$ ) and medium frequency over mitral area (ma mf) showing the pansystolic murmur, opening snap, and third heart sound. Also shown are electrocardiogram carotid and respiratory traces. (b) This shows in addition an apex cardiogram (acg) with the outward rapid filling wave (ORFw). $\mathrm{a}=a$ wave. 
gery. One occurred suddenly at 2 months, the patient having been previously well, and was possibly due to dysrhythmia. One patient died at 27 months while undergoing a second mitral valvar replacement, and in I patient, who died 14 months after operation, the cause of death was not established. The mitral valve was slightly incompetent and the histology is discussed later.

Only one of these late deaths can with certainty be attributed to failure of the fascia lata valve in that its malfunction required reoperation which ended in death. Unfortunately necropsy data on the others are inadequate to be certain of the role of mitral valvar dysfunction.

\section{Mitral regurgitation}

Of the $4 \mathrm{I}$ survivors, $37(90 \%)$ left hospital with clinically competent mitral valve but only 2 of these valves remain competent as assessed in July 1973.
Five patients have had to have their fascia lata valves replaced by prosthetic valves and a further 9 patients are unwell and some of these will probably also require further operation. Eighteen patients have clinical evidence of mitral regurgitation, not severe enough to produce serious symptoms, but the clinical features show that it is progressive.

Despite careful follow-up, the rate of development of mitral regurgitation has been difficult to document. This is explained by the difficulty in assessing the importance of a systolic murmur heard in outpatients, especially in the first few months after operation before we were aware of the natural history of this valve. However, at I year, I4 of the 37 survivors $(38 \%)$ had auscultatory evidence of mitral regurgitation, and the characteristic features of such a leaking valve are shown in Fig. I. These signs have generally been followed by increasing heart size and symptomatic deterioration. The

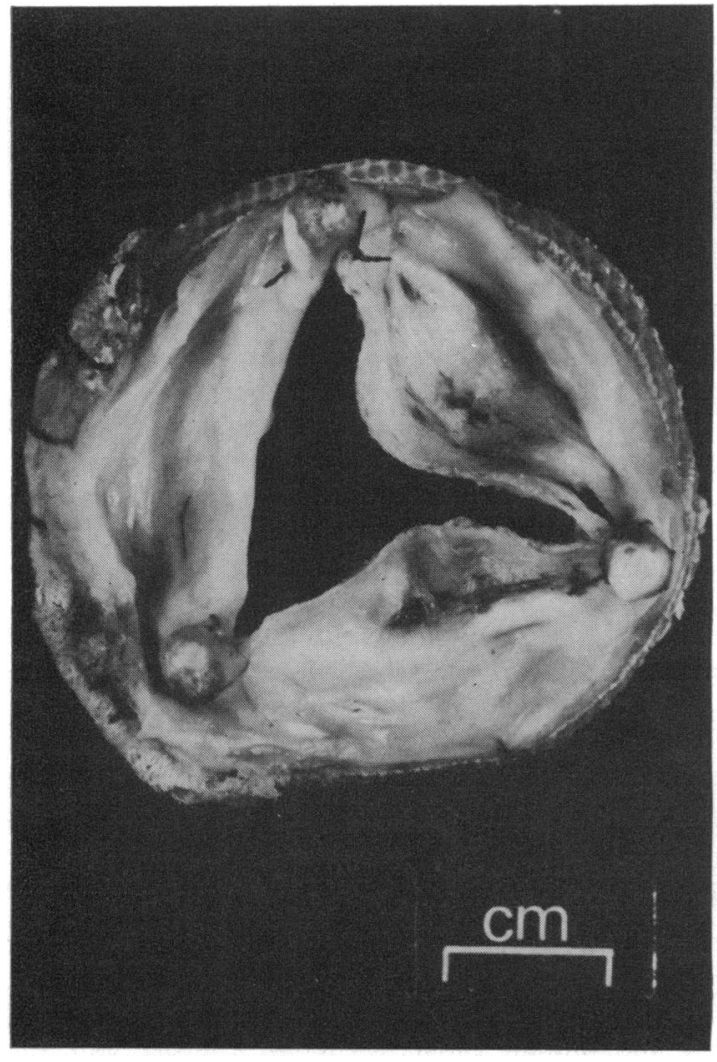

$a$

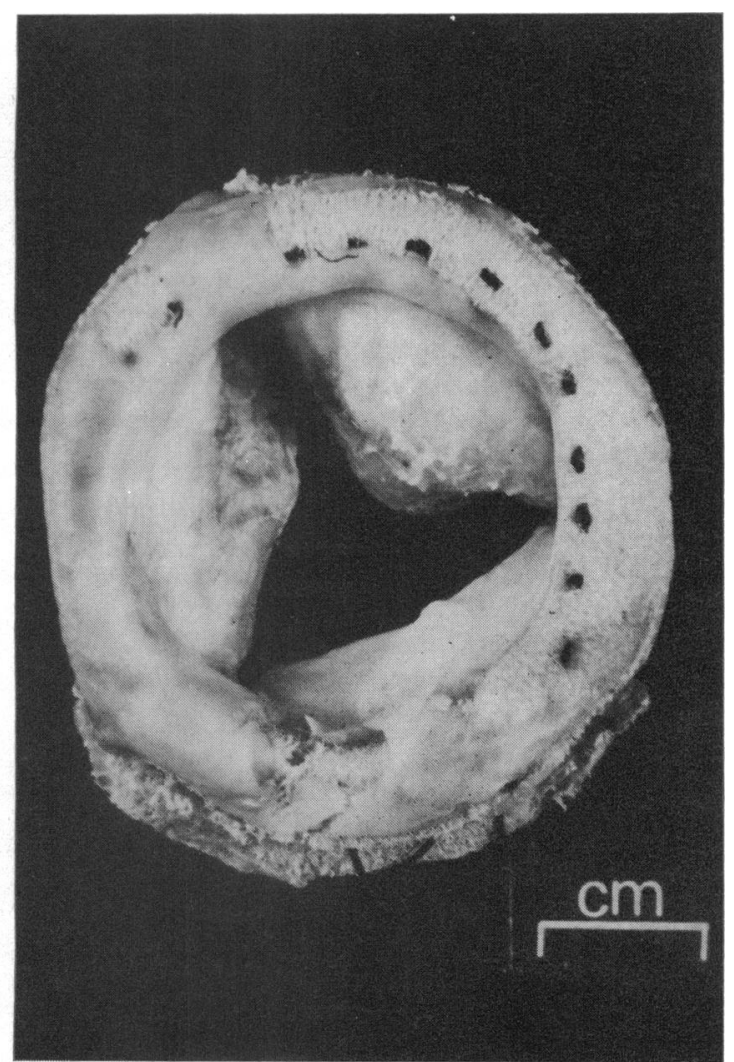

$b$

FIG. 2 Macroscopical appearance of a fascia lata valve removed 27 months after operation showing the typical retraction and thickening of the posterior cusps. (a) Ventricular aspect; (b) atrial aspect. 
deterioration has been slow and relentless and none has developed regurgitation acutely.

\section{Reinvestigation}

Twenty-two patients had postoperative cardiac catheterization though not all parameters were measured in all patients. Thirteen had a small resting end-diastolic mitral valvar gradient (0-5 $\mathrm{mmHg}$ ). In 4 patients the gradient varied between 4 and $10 \mathrm{mmHg}$ and in I patient the gradient exceeded Io mmHg. The pulmonary artery pressure was normal in 7 and remained raised in 15 .

Left ventricular angiography was performed in 19 patients and the mitral valve was competent at the time of study in 5. Eight had mild, 4 moderate, and 2 severe mitral regurgitation.

Three patients have been reinvestigated on two separate occasions. In all 3 the resting pressures and indirect mitral gradients were similar at both studies. Left ventricular angiography revealed mitral regurgitation in all patients at the second study, while I of the 3 had had a competent valve at the first study. In I patient the degree of regurgitation was unchanged and in $I$ it had become more severe.

\section{Endocarditis}

There have been no cases of postoperative endocarditis, and only I patient had had an attack before operation. Patients had their teeth carefully examined before surgery to eliminate sepsis and all survivors remain under close supervision, and have penicillin prophylaxis for all dental procedures.

\section{Embolism}

The incidence of postoperative embolism has been low. Systemic embolism has occurred after operation in 2 patients causing death in $I$ at 3 days and leg ischaemia in the other at 3 years. This second patient was not taking anticoagulants and was in atrial fibrillation at the time.

\section{Pathology}

Five mitral fascia lata valves have been removed at operation with I death and a further 13 patients have died. The pathology of these valves has been described (McEnany et al., 1972) and the macroscopical appearance of one such valve is shown in Fig. 2. The cusps are thickened, shrunken, and retracted and this process is most pronounced in the posterior cusps opposite the aortic root. In the patient who died at 14 months the changes were characteristic but the anterior cusp was thin, flexible, and competent while the posterior ones were thick and immobile. In addition the sinus behind the posterior cusp was partly filled with organized thrombus and there was a thin layer of thrombus partly surrounding the upper surface of the ring and adjacent endocardium.

The histology of this valve revealed excessively dense collagen with fissuring and fragmentation which could not be attributed to a processing artefact. Histology of another valve showed that the central part of the anterior cusp was dense acellular tranversely oriented collagen bundles. On either side of this central area, there were thin layers of cellular collagen running longitudinally over the surface. Mucopolysaccharide-like metachromasia was shown in the outer layers, and on the ventricular. surface near the periphery there was some elastic tissue and two small thrombosed muscular arteries. Silver staining showed reticulin only in the longitudinal outer layers.

In the periphery of the posterior cusp there was $2 \mathrm{~mm}$ of compact mature bone with irregular ossification lines and cellular lacunae with osteoblastic activity in the outermost edge. The rest of the leaflet looked like the anterior cusp.

Even though this was living tissue at the time of insertion, and viability studies in two valves removed in the first year showed the presence of cells, no living cells have been seen in any fascial valve removed after 2 years (N. Al-Janabi, personal communication).

\section{Discussion}

The early promise of autologous fascia lata for mitral valvar replacement has not been fulfilled. Fascia lata valves were initially satisfactory and 90 per cent of our patients left hospital with no evidence of valvar dysfunction. However, at the time of the present study only 2 out of 37 patients appear to have clinically competent valves.

The auscultatory features of mitral regurgitation tend to appear after the first 6 to 12 months and the lesion becomes progressively more severe, though the rate of deterioration is variable. The progressive nature of the regurgitation has been confirmed by serial angiographic studies in 2 patients and by clinical observation in many. The auscultatory and haemodynamic findings show that the regurgitation is associated with some stenosis which is due to stiffening of the valve cusps. Eventually within I to 3 years the regurgitation becomes haemodynamically important, requiring further valve replacement. It seems unlikely that any of these valves will be functioning satisfactorily at 5 years and the majority will probably have to be replaced.

Not all patients have been restudied by cardiac catheterization. However, when it became apparent that these valves were not functioning satisfactorily 
it seemed important to document this with haemodynamic and angiographic studies. Because of this, and because some patients have been reinvestigated with a view to further operation, haemodynamic and angiographic data are now available in 22 of the survivors. All of these patients have shown a mitral diastolic valvar gradient at rest (pulmonary artery wedge pressure compared with left ventricular end diastolic pressure) though this was usually small. There was angiographic incidence of mitral regurgitation in $13(72 \%)$. The angiographic incidence of mitral regurgitation is less than the clinical incidence $(88.3 \%)$, but this is explained by the fact that the lesion is progressive and the mean followup period for the former (13.8 months) is shorter than the latter ( 33.7 months).

The clinical and investigatory findings suggest that fascia lata valves in the mitral position become both shrunken and rigid, and this is confirmed by the observed pathology findings. As has been described (McEnany et al., 1972), the cusp degeneration affects primarily the posterior cusps leaving the anterior cusp relatively well preserved, which is of interest because of the importance of the anterior leaflet in the normal mechanism of mitral valve closure (Reid, 1969). The superior results obtained by the use of autologous fascia lata in aortic valvar replacement (Joseph et al., 1973) and the disappointing results from its use in the reconstruction of the outflow tract of the right ventricle (Ross and Somerville, I97I) may suggest that the greater the pressures imposed on fascia lata the better it performs (Ross et al., 1972). It has so far not been possible to identify any obvious preoperative or operative factors that might separate the best from the worst results, and it remains possible that there is some individual variation in the quality of the fascia lata. It is also possible that there have been variations in the technical skill involved in making the valve as several different surgeons constructed them.

The theoretical advantages of using biological materials for valvar replacement remain (Ross, 1967). These advantages cannot, however, compensate for the progressive failure of the autologous fascia lata in the replacement of the mitral valve position. We have abandoned this tissue in favour of inverted aortic homografts or prosthetic valves in the mitral position. The homograft valves retain the advantages of a biological valve and are already giving superior long-term results (Oh et al., 1973; Yacoub, Towers, and Somerville, 1972).

The experience with fascia lata illustrates the pitfalls of an early and optimistic assessment of a new valve whether mechanical or biological. Early reports (Ionescu and Ross, 1969) suggested good results at 6 to 12 months. However, there were indications after the first year that the optimism for the mitral valve might not be justified so the technique was abandoned in the National Heart Hospital until critical analysis over a longer period could be made. Subsequent experience has confirmed the view that autologous fascia lata is unsuitable for use as a biological valve substitute.

We thank the physicians who have allowed us to study their patients, including Dr. G. Hayward, Dr. W. Brigden, Dr. A. Leatham, and Professor P. Harris. We are indebted to Dr. Al-Janabi of the Homograft Department of this hospital and to Dr. R. Sutton for the phonocardiogram, illustrated in Fig. I. We are most grateful to Dr. Luis Becu for interpretation of the histological findings.

\section{References}

Ionescu, M. I., and Ross, D. N. (1969). Heart-valve replacement with autologous fascia lata. Lancet, $2,335$.

Ionescu, M. I., Ross, D. N., Wooler, G. H., Deac, R., and Ray, D. (1970). Replacement of heart valves with autologous fascia lata. British fournal of Surgery, 57, 437.

Joseph, S., Somerville, J., Emanuel, R., Ross, D., Ross, K., and McDonald, $L$. Aortic fascia lata valves. In preparation.

McEnany, M. T., Ross, D. N., and Yates, A. K. (1972). Cusp degeneration in frame-supported autologous fascia lata mitral valves. Clinical results in 67 patients. Thorax, 27, 23.

Oh, W., Somerville, J., Ross, D. N., Ross, K. J., and Emanuel, R. (1973). Mitral valve replacement with preserved cadaveric aortic homografts. Fournal of Thoracic - and Cardiovascular Surgery, 65, 712.

Reid, K. G. (1969). Mitral valve action and the mode of ventricular filling. Nature (London), 223, 1383.

Ross, D. N. (1967). Homograft replacement of the aortic valve technique. British fournal of Surgery, 54, 165.

Ross, D. N., Gonzalez-Lavin, L., and Dalichau, H. (1972). Two-year experience with supported autologous fascia lata for heart valve replacement. Annals of Thoracic Surgery, 13, 97.

Ross, D. N., and Somerville, J. (1971). Fascia-lata reconstruction of the right ventricular outflow tract. Lancet, 1, 94I.

Senning, A. (1967). Fascia lata replacement of aortic valves. fournal of Thoracic and Cardiovascular Surgery, 54, 465.

Yacoub, M., Towers, M., and Somerville, W. (1972). Mitral valve replacement using fresh 'unstented' semilunar valve homografts (abstract). British Heart fournal, 34, 207.

Requests for reprints to Dr. Jane Somerville, Cardiothoracic Institute, 2 Beaumont Street, London WIN 2DX. 